\title{
Anatomía quirúrgica del drenaje venoso en la región del triángulo carotídeo*
}

\author{
Drs. JOSÉ GONZÁLEZ R. ${ }^{1}$, MARIO CANTÍN L. ${ }^{2}$, CÉSAR CORONADO G. ${ }^{2}$, IVÁN SUAZO G. ${ }^{2}$
}

1 Cirujano Dentista, Práctica Privada.

2 Departamento de Morfología, Facultad de Ciencias de la Salud, Universidad de Talca. Talca, Chile.

\begin{abstract}
\section{Surgical anatomy of jugular vein tributaries}

Background: The main tributaries of the internal jugular vein join at cervical level through the venous thyrolinguofacial trunk. This trunk is classically described as formed by the union of the facial, superior thyroid and lingual veins. Aim: To evaluate variations in the formation of the thyrolinguofacial trunk in human cadavers. Material and Methods: Thirty hemi-necks were dissected in human cadavers of nine men and six women, with ages ranging between 29 and 50 years, and analyzed by direct observation. Results: In 16 hemi-necks $(53.3 \%)$, the trunk was thyrolinguofacial; in seven $(23.3 \%)$, it was linguofacial; in six $(20 \%)$, it was thyrolingual and in one case $(3.3 \%)$ it was thyrolinguo pharyngofacial. No thyrofacial trunk formation was found. There was a correlation between the diameter of the internal jugular vein and of the venous trunk formed by these veins. Conclusions: There are morphological changes in the formation patterns of facial, lingual and superior thyroid veins. This information is useful for surgical oncology, plastic surgery, head and neck surgery and radiology.
\end{abstract}

Key words: Thyrolinguofacial trunk, facial vein, superior thyroid vein, lingual vein.

\section{Resumen}

Los principales afluentes de la vena yugular interna se incorporan a nivel cervical por medio del tronco venoso tirolinguofacial. Clásicamente se ha descrito su formación por la unión de la vena facial, lingual y tiroidea superior. El objetivo de este estudio fue determinar las variaciones en la presentación del tronco tirolinguofacial en cadáveres humanos de la región del Maule, Chile. Se disecaron 30 hemicuellos pertenecientes a 15 cadáveres humanos de ambos sexos, con un rango de edad entre 29 y 50 años, y se analizaron mediante observación directa. Se observó la formación de tronco venoso en la totalidad de los casos, siendo $53,3 \%$ correspondiente al tronco de tipo tirolinguofacial, 23,3\% linguofacial, 20\% tirolingual y sólo un 3,3\% el tronco tirolinguofaringofacial. No se encontró la formación del tronco venoso de tipo tirofacial. Además se encontró una correlación significativa entre el diámetro de la vena yugular interna y el tronco venoso conformado por estas venas. Por lo tanto, existen variaciones morfológicas en los patrones de conformación de las venas facial, lingual y tiroidea superior, siendo estos datos de importancia para áreas de cirugía oncológica, cirugía plástica, cirugía de cabeza y cuello y radiología.

Palabras clave: Tronco tirolinguafacial, vena facial, vena tiroidea superior, vena lingual.

*Recibido el 17 de Agosto de 2009 y aceptado para publicación el 24 de Noviembre de 2009.

Correspondencia: Dr. Iván Suazo G.

Avenida Lircay s/n. Of.104, Talca, Chile.

E-mail: isuazo@utalca.cl 


\section{Introducción}

El sistema venoso debido a su complejo desarrollo embriológico a menudo da lugar a variaciones clínicamente relevantes ${ }^{1}$. Estas variaciones son una característica común del sistema vascular ${ }^{2}$, siendo más común en las venas que en las arterias ${ }^{3}$.

La vena yugular interna es una gran vena que recoge la mayor parte de la sangre de la cabeza y el cuello. Se inicia como una continuación del seno sigmoideo en la base del cráneo, se extiende verticalmente hacia abajo en el cuello y se une a la vena subclavia para formar la vena braquiocefálica ${ }^{4}$. En el cuello y en las proximidades del hueso hioides, la vena yugular interna recibe sus afluentes más importantes, que son las venas facial, lingual, tiroidea superior, faríngea y tiroidea media. En la mayor parte de los casos, las tres primeras de estas colaterales desembocan en la vena yugular interna por medio de un tronco tirolinguofacial (TTLF) ${ }^{5}$.

El tronco tirolinguofacial es corto y voluminoso, el cual se forma por la unión de la vena facial, lingual y tiroidea superior, cruza la carótida común cerca de su bifurcación o a nivel de ésta. Desemboca drenando en la vena yugular interna frente al borde superior del cartílago tiroides, o un poco superiormente, a la altura del espacio tirohioideo ${ }^{5}$. Además puede tener otra afluente inconstante, la vena faríngea, procedente de una red venosa formada en la pared de la faringe, suelen ser dos o tres venas que desembocan a diferentes alturas en la vena yugular interna o en algunas ocasiones en el tronco tirolinguofacial ${ }^{6}$, entonces en estos casos se convierte en tronco tirolinguofaringofacial ${ }^{7-10}$.

Sin embargo, esta disposición puede cambiar, las venas tiroidea superior y lingual se pueden unir formando independientemente el tronco venoso tirolingual. Entre la unión de las venas facial y lingual se origina el tronco linguofacial y el tronco tirofacial, que resulta de la unión de las venas tiroidea superior y facial, y que son encontrados en la literatura con una frecuencia entre 2 y $6,9 \%{ }^{11}$.

El objetivo de este estudio fue describir las variaciones anatómicas en los patrones de formación de troncos venosos originados a partir de las venas facial, lingual y tiroidea superior en cadáveres humanos en la VII región del Maule, Chile.

\section{Material y Método}

La muestra analizada correspondió a 30 caras laterales de cuello pertenecientes a 15 cadáveres humanos adultos de ambos sexos (9 hombres y 6 mujeres), no fijados, mantenidos en cámaras frigoríficas a $0^{\circ}$ grados Celsius, a los cuales se les realizó la autopsia de rigor junto al médico tanatólogo, proce- diéndose al análisis del tronco tirolinguofacial. Las muestras fueron obtenidas desde el Servicio Médico Legal (SML) de Curicó, Chile, y correspondieron a fallecidos no reclamados. Toda la autorización necesaria fue gestionada con el SML.

Las regiones cervicales fueron disecadas identificando las venas facial, lingual y tiroidea superior y los troncos venosos que ellas formaban.

Para realizar el acceso, el cadáver se posicionó decúbito supino con el fin de realizar la disección anterolateral en la zona cervical. Se realizó una incisión continua en la zona mediana cervical, desde el borde basilar mandibular, seguida de una descarga recta en sentido vertical y caudal hasta el límite superior del esternón. Posteriormente se realizaron dos incisiones horizontales bilaterales paralelas entre sí, una superior en relación al borde basilar y otra inferior en relación al borde superior del esternon (Figura 1).

Se accedió a la zona rechazando sucesivamente la piel, lámina superficial y músculo platisma, lámina de revestimiento de la fascia profunda junto con el músculo esternocleidomastoideo (desde su inserción esternal) y la lámina pretraqueal con el músculo homohioideo para luego observar la constitución del tronco y sus variaciones anatómicas del lado derecho e izquierdo (Figura 2).

Una vez realizada la clasificación del tronco constituido, se procedió a la medición del diámetro del tronco tirolinguofacial o la variación anatómica que se haya constituido mediante la utilización de un Caliper digital $(0,01 \mathrm{~mm})$, una medición, realizada por un solo operador, tomando como punto constante de medición del tronco conformado un punto ubicado a $5 \mathrm{~mm}$ de distancia de la unión de este con la vena yugular interna.

En los casos en que no se formó ningún tipo de tronco venoso y, los elementos venosos se encuentren de manera independiente, se obtuvo la medida de la vena de mayor calibre. Del mismo modo, se realizó la medición del diámetro de la vena yugular interna a una distancia de $5 \mathrm{~mm}$ de la llegada del tronco venoso constituido.

Posteriormente, mediante el uso de una regla milimetrada se determinó la distancia existente entre el tronco venoso constituido en la zona, tomando como referencia de medición el vértice inferior formado por la unión del tronco venoso con la vena yugular interna, hasta el tronco venoso braquiocefálico del lado correspondiente (tronco venoso braquicefálico derecho o izquierdo).

Se realizó el análisis estadístico descriptivo de los resultados obtenidos y se calculó la significancia estadística aplicando la prueba $\mathrm{T}$ de Student con $\mathrm{p}<0,01$ utilizando el software SPSS 15.0 para Windows XP. 


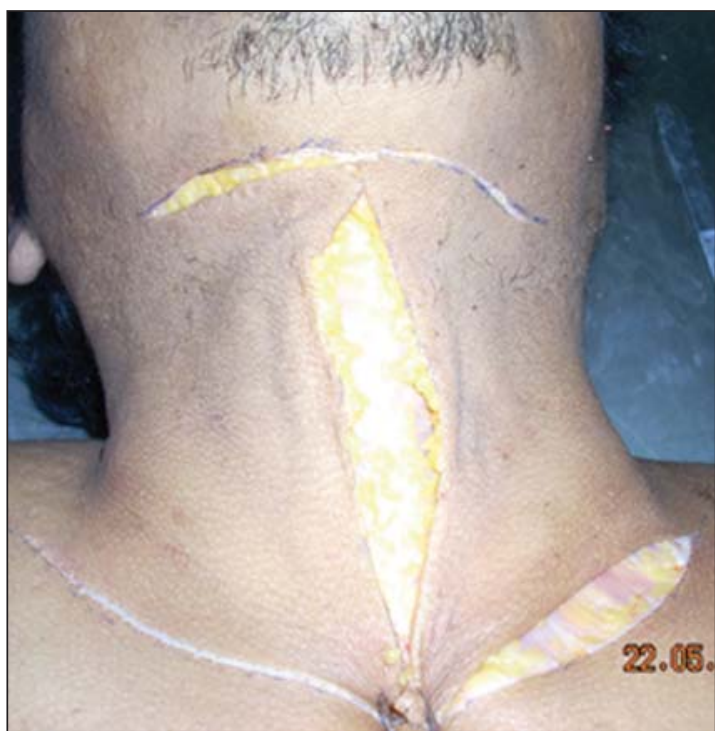

Figura 1. Esquema de las incisiones realizadas con una incisión vertical en la línea mediana y dos horizontales en los límites superior e inferior de la región cervical.

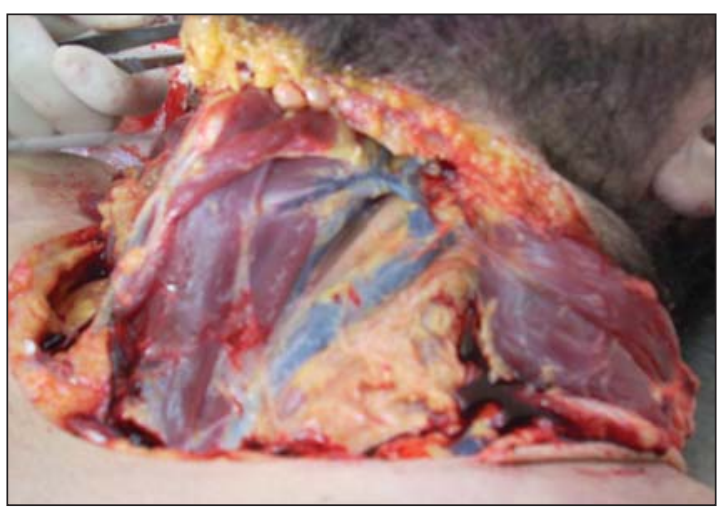

Figura 2. Acceso y visualización del tronco venoso luego del rechazo de las estructuras superficiales.

\section{Resultados}

Al analizar la constitución de los troncos venosos conformados en la zona cervical en el total de la muestra de hemicuellos estudiada $(\mathrm{n}=30)$, se determinó que el tronco conformado con mayor frecuencia en ambos lados correspondió al tronco venoso Tirolinguofacial en el 53,3\% de los casos (16 hemicuellos), el tronco venoso linguofacial se presentó en el 23,3\% (7 hemicuellos), el tronco venoso de tipo tirolingual se presentó en el $20 \%$ de la muestra (6 hemicuellos). En un solo caso (3,3\%, hemicuello izquierdo) se encontró que la constitución del tronco venoso fue de tipo tirolinguofaringofacial. En ninguno de los hemicuellos analizados se encontró conformación de tronco venoso de tipo tirofacial (Tabla 1, Figura 3).

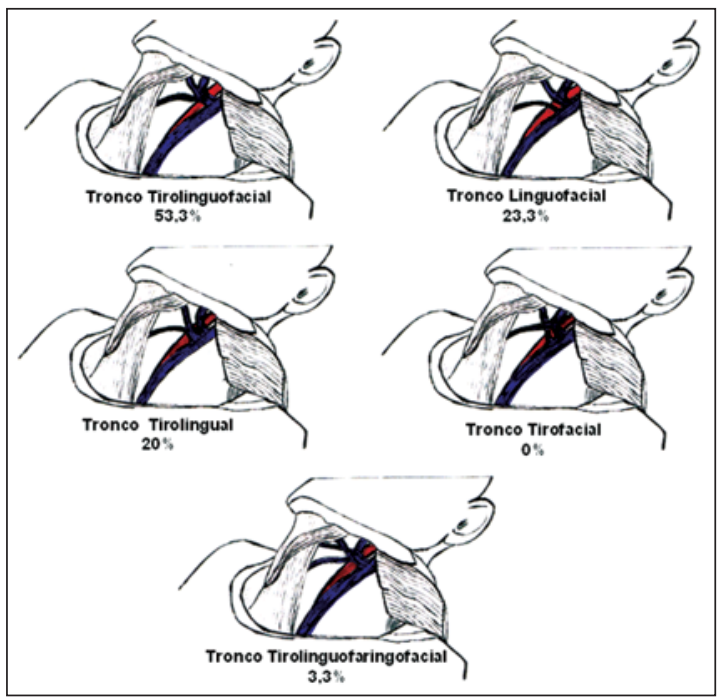

Figura 3. Representación esquemática de la frecuencia de formación del tronco venoso en el total de la muestra $(\mathrm{n}=30)$.

Tabla 1. Frecuencia y porcentaje de presentación de los troncos venosos conformados en una muestra de 30 hemicuellos

\begin{tabular}{lccccc}
\hline \multicolumn{2}{l}{ Tronco venoso } & Frecuencia & Porcentaje & Porcentaje válido & Porcentaje acumulado \\
\hline Válidos & TLF & 16 & 53,3 & 53,3 & 53,3 \\
& LF & 7 & 23,3 & 23,3 & 76,7 \\
& TL & 6 & 20,0 & 20,0 & 96,7 \\
& TLFF & 1 & 3,3 & 3,3 & 100,0 \\
& Total & 30 & 100,0 & 100,0 & \\
\hline
\end{tabular}

TLF: Tirolinguofacial, LF: Linguofacial, TL: Tirolingual, TLFF: Tirolinguofaringofacial. 
Tabla 2. Diámetro de los troncos venoso según el tipo de tronco que se conformó

\begin{tabular}{llcccccccccc}
\hline & & \multicolumn{1}{c}{ Diámetro del tronco (mm) } & \multicolumn{3}{c}{ Total } \\
& & $\mathbf{3 , 0 0}$ & $\mathbf{4 , 0 0}$ & $\mathbf{5 , 0 0}$ & $\mathbf{5 , 5 0}$ & $\mathbf{6 , 0 0}$ & $\mathbf{7 , 0 0}$ & $\mathbf{8 , 0 0}$ & $\mathbf{9 , 0 0}$ & $\mathbf{1 0 , 0 0}$ & \\
\hline \multirow{2}{*}{ Tronco } & TLF & 0 & 0 & 2 & 1 & 0 & 2 & 5 & 2 & 4 & 16 \\
& LF & 1 & 3 & 0 & 0 & 1 & 1 & 1 & 0 & 0 & 7 \\
& TL & 1 & 3 & 2 & 0 & 0 & 0 & 0 & 0 & 0 & 6 \\
& TLFF & 0 & 0 & 0 & 0 & 0 & 0 & 1 & 0 & 0 & 1 \\
\hline
\end{tabular}

TLF: Tirolinguofacial, LF: Linguofacial, TL: Tirolingual, TLFF: Tirolinguofaringofacial.

Tabla 3. Diámetro de la vena yugular interna según el tipo de tronco conformado

\begin{tabular}{llcccccccccccc}
\hline & \multicolumn{1}{c}{ Diámetro de la vena yugular interna (mm) } & \multicolumn{1}{c}{ Total } \\
& & $\mathbf{1 4 , 0 0}$ & $\mathbf{1 5 , 0 0}$ & $\mathbf{1 6 , 0 0}$ & $\mathbf{1 7 , 0 0}$ & $\mathbf{1 8 , 0 0}$ & $\mathbf{1 9 , 0 0}$ & $\mathbf{2 0 , 0 0}$ & $\mathbf{2 1 , 0 0}$ & $\mathbf{2 2 , 0 0}$ & $\mathbf{2 3 , 0 0}$ \\
\hline \multirow{2}{*}{ Tronco } & TLF & 0 & 0 & 1 & 3 & 1 & 4 & 2 & 2 & 2 & 1 & 16 \\
& LF & 0 & 3 & 1 & 0 & 2 & 0 & 0 & 0 & 0 & 1 & 7 \\
& TL & 2 & 2 & 1 & 1 & 0 & 0 & 0 & 0 & 0 & 0 & 6 \\
& TLFF & 0 & 0 & 0 & 0 & 0 & 1 & 0 & 0 & 0 & 0 & 1 \\
Total & & 2 & 5 & 3 & 4 & 3 & 5 & 2 & 2 & 2 & 2 & 30 \\
\hline
\end{tabular}

TLF: Tirolinguofacial, LF: Linguofacial, TL: Tirolingual, TLFF: Tirolinguofaringofacial.

De acuerdo a la medición del diámetro de los troncos venosos conformados, estos presentaron un diámetro variable entre $3 \mathrm{~mm}$ y $10 \mathrm{~mm}$ con una media de $6,55 \mathrm{~mm}$ y una desviación estándar de 2,27. En la Tabla 2 se observa el detalle del tipo de tronco y su diámetro en cada caso (Tabla 2).

De acuerdo a la medición del diámetro de la vena yugular interna, se encontró un diámetro variable entre $14 \mathrm{~mm}$ y $23 \mathrm{~mm}$, con una media de $18 \mathrm{~mm}$ y una desviación estándar de 2,66. En la Tabla 3 se observa la relación según tipo de tronco y diámetro de la vena yugular interna. Al analizar la relación entre el diámetro de la vena yugular interna y el tronco venoso conformado se encontró una correlación significativa entre ellos $(\mathrm{p}<0,01)$. No se encontraron diferencias significativas $(\mathrm{p}=0,446)$ con respecto a los valores encontrados en las mediciones del lado derecho e izquierdo de los cadáveres estudiados.

\section{Discusión}

El tronco venoso encontrado con mayor frecuencia fue el tronco venoso tirolinguofacial, el cual se presentó en el 53,3\% de la muestra analizada. Estos resultados muestran una mayor frecuencia a la reportada por Gusmão et $\mathrm{al}^{11}$, quienes describen en $\mathrm{su}$ estudio que el $38,1 \%$ del total de los casos corres- pondían a troncos venosos de tipo tirolinguofacial; Bruno y Ghezzi (citado por Gusmão et $\mathrm{al}^{11}$ ) lo informan en el 30\% de los casos analizados y Madeira (citado por Gusmão et $\mathrm{al}^{11}$ ) lo encontraron tan sólo en el $10 \%$ de las muestras estudiadas.

El segundo más frecuente fue el tronco venoso linguofacial presentándose en un 23,3\% $(n=7)$. Similares a los resultados de Gusmão et al ${ }^{11}$, quienes lo encontraron en un $14,2 \%$. La conformación de este tipo de tronco era esperable, debido a que en un embrión humano de $10 \mathrm{~mm}$ se encuentra la vena faríngea ventral, la que posteriormente en un embrión de $18 \mathrm{~mm}$ se denomina vena linguofacial ${ }^{12}$, la que se desarrolla de manera independiente pudiendo o no incluir más elementos, por lo que este tipo de formación venosa tiene una fuerte correlación embriológica.

El tronco venoso tirolingual se presentó en un $20 \%$ de los casos analizados $(n=6)$ en contraste a lo encontrado por Gusmão et al $^{11}$, quienes lo describen en un $4,8 \%$ de los casos, presentando este la exclusión en su conformación de la vena facial, la cual se presenta como una afluente de la vena yugular interna de manera aislada. Se ha descrito el drenaje de la vena facial en diferentes venas superficiales, Kopuz et al (citado por Prakash ${ }^{1}$ ), describen un curso inusual de la vena facial derecha, la cual se unía 
a nivel intraparotídeo a la vena retromandibular y terminaba uniéndose a la vena temporal superficial derecha. Por otro lado, Choundhry et al ${ }^{12}$, encontraron que la terminación de la vena facial separada del tronco en la vena yugular externa ocurre en el 5\% de los individuos; Gupta et al ${ }^{13}$, describieron que en $16(9 \%)$ de 178 caras anterolaterales de cuello la vena facial terminaba en la vena yugular externa. Prakash et $\mathrm{al}^{1}$, durante una disección de cabeza y cuello en un cadáver adulto masculino, descubrieron un inusual patrón de drenaje de la vena facial donde ésta se unía a la división anterior de la vena retromandibular para formar la vena facial común y ésta a su vez terminaba en la vena subclavia izquierda. En este estudio no se encontró ningún patrón de drenaje poco común en relación a la vena facial, sin embargo, se necesita una mayor cantidad de muestras para obtener resultados cercanos a los autores anteriormente citados.

El tronco venoso tirolinguofaringofacial se presentó en nuestra muestra con la menor frecuencia, la cual fue tan sólo de 3,3\%, al igual que Gusmão et $\mathrm{al}^{11}$, quienes lo encontraron en $2,4 \%$.

La variabilidad en la conformación de los troncos venosos originados por la vena facial, lingual y tiroidea superior puede explicarse por su desarrollo, ya que durante la embriogénesis del sistema venoso, la mayoría de las venas se desarrollan a partir de plexos capilares ${ }^{11,14}$, los que inicialmente aumentan en complejidad por brotes (angiogénesis) y anastomosis, para luego fusionarse y crecer, originando escasos y largos vasos, muchos de los cuales son transitorios y desaparecen ${ }^{15}$.

Los diámetros más amplios encontrados fueron en los troncos venosos conformados por una mayor cantidad de venas como por ejemplo el tronco tirolinguofacial y tirolinguofaringofacial, por el contrario el diámetro menor correspondió al tronco venoso de tipo tirolingual el cual presentó diámetros entre 3 y $5 \mathrm{~mm}$, lo que se explicaría directamente por la cantidad de sangre venosa que drenan dichos vasos según el territorio que les corresponde.

Según el tipo de tronco venoso conformado, los diámetros encontrados en la vena yugular interna fueron variables, existiendo una correlación significativa entre ellos ( $p<0,01)$, por lo tanto, mientras mayor sea el diámetro del tronco conformado, mayor será el diámetro de la vena yugular interna. Los resultados encontrados en nuestro estudio son muy relevantes para la cirugía microvascular de reconstrucciones complejas de la cavidad oral mediante colgajos miocutáneos, porque al existir un buen calibre de las venas es más fácil para el cirujano llevar a cabo la creación de microanastomosis en los pedículos quirúrgicos y al mismo tiempo se reduce el tiempo de la cirugía con una menor morbilidad. Al llevar a cabo estos procedimientos se privilegian estructuras vasculares con un calibre adecuado como el tronco tirolinguofacial o la vena yugular externa en desmedro de tronco venosos de menor calibre ${ }^{16}$.

\section{Referencias}

1. Prakash BB, Bhagath KP. A rare termination of left common facial vein into the left subclavian vein. A case report. Int J Morphol 2007; 25: 555-556.

2. Peuker ET, Ficher G, Filler TJ. Correspondence-facial vein terminating in the superficial temporal vein: a case report. J Anat 2001; 198: 509-510.

3. D'Silva SS, Pulakunta T, Potu BK. Termination of the facial vein into the external jugular vein: an anatomical variation. J Vasc Bras 2008; 7: 174-175.

4. Nayak BS. Surgically important variations of the jugular veins. Clin Anat 2006; 19: 544-546.

5. Rouvière H, Delmas A. Anatomía Humana; Descriptiva, Topográfica y Funcional, Tomo I. 10 ed. Barcelona, Editorial Masson, 1999: 215-220.

6. García-Porrero JA, Hurlé JM. Anatomía humana. Madrid, McGraw-Hill Interamericana, 2005: 185-189.

7. Latarjet M, Ruiz-Liard DA. Anatomía Humana. $4^{\mathrm{a}}$ ed. Buenos Aires, Editorial Panamericana, 2004: 10401043.

8. Gray H. Anatomy of the human body. Philadelphia, Lea e Febiger, 1985: 546-548.

9. Testut L, Jacob C. Tratado de Anatomía Topográfica con aplicaciones médico quirúrgicas. $8^{\mathrm{a}}$ ed. Barcelona, Editorial Científico-Médica, 1958: 312-319.

10. Pauchet V, Dupret S. Atlas de Anatomia. $5^{\mathrm{a}}$ ed. Río de Janeiro, Atheneu, 1965: 130-132.

11. Gusmão LCB, Sousa-Rodrigues CF, Nóbrega FSG, Pereira Da Silva LMT. Drenaje de las venas facial, lingual y tiroidea superior en el hombre. Int J Morphol 2006; 24: 685-688.

12. Choudhry R, Tulia A, Choudhry S. Facial vein terminating in the external jugular vein. An embriologic interpretation. Surg Radiol Anat 1997; 19: 73-77.

13. Gupta V, Tuli A, Choudhry R, Agarwal S, Mangal A. Facial vein into external jugular vein in humans: its variations, philogenitic retention and clinical relevance. Surg Radiol Anat 2003: 25: 36-41.

14. Hamilton WJ, Boyd JD, Mossman HW. Embriología Humana. $4^{\mathrm{a}}$ ed. Buenos Aires, Editorial Intermédica, 1973: 279-287.

15. Arey LB. Anatomía del Desarrollo. $4^{\mathrm{a}}$ ed. Barcelona, Editorial Vásquez, 1962: 308-310.

16. Dean Ferrer A, Alamillos FJ, Redondo Camacho A, Torres Corpas J, Ruiz Masera JJ, Zafra Camacho F, y col. Reconstrucción compleja de la cavidad oral mediante dos colgajos simultáneos de peroné y radial. Rev Esp Oral \& Maxilofac 2004; 26: 306-315. 